\title{
Anti-apoptotic hPEBP4 silencing promotes TRAIL-induced apoptosis of human ovarian cancer cells by activating ERK and JNK pathways
}

\author{
PENG LI ${ }^{1,2^{*}}$, XIAOJIAN WANG ${ }^{3 *}$, NAN LI $^{2}$, HUABEI KONG ${ }^{1}$, ZHENHONG GUO $^{2}$, \\ SHUXUN LIU ${ }^{2}$ and XUETAO CAO ${ }^{2,3}$ \\ ${ }^{1}$ Department of Obstetrics and Gynecology, Qilu Hospital of Shandong University, Shandong, \\ Jinan 250012; ${ }^{2}$ Institute of Immunology, Second Military Medical University, Shanghai 200433; \\ ${ }^{3}$ Institute of Immunology, Tsinghua University, Beijing 100084, P.R. China
}

Received March 20, 2006; Accepted May 17, 2006

\begin{abstract}
Tumor necrosis factor-related apoptosis-inducing ligand (TRAIL) induces apoptosis in a variety of tumor cell lines but not typically in normal or nontransformed cells, which makes TRAIL a desirable therapeutic agent to fight cancer. Human phosphatidylethanolamine-binding protein 4 (hPEBP4) is a recently identified anti-apoptotic molecule and has been shown to be highly expressed in breast and ovarian cancer cells. We demonstrate that silencing of hPEBP4 in CaoV-3 ovarian cancer cells potentiates TRAIL-induced apoptosis. We found that endogenous hPEBP4 interacts with Raf-1 and MEK1 in TRAIL-treated CaoV-3 cells by coimmunoprecipitation analysis. Simultaneously, silencing of hPEBP4 in CaoV-3 cells enhances TRAIL-induced ERK and JNK activation. Moreover, the inhibitors of MEK1 or JNK can reduce hPEBP4-silence-induced TRAIL sensitivity. Therefore, silencing of hPEBP4 in CaoV-3 ovarian cancer promotes TRAIL-induced apoptosis, and the increased MAPK activation is required for the apoptosis sensitization. All these data indicate that silencing of hPEBP4, an important potential
\end{abstract}

Correspondence to: Dr Xuetao Cao, Institute of Immunology, Second Military Medical University, 800 Xiangyin Road, Shanghai 200433, P.R. China

E-mail: caoxt@public3.sta.net.cn

Abbreviations: ERK, extracellular signal-regulated kinase; MEK, extracellular signal-regulated kinase kinase; PI, propidium iodide; PEBP, phosphatidylethanolamine binding protein; PE, phosphatidylethanolamine; TRAIL, tumor necrosis factor-related apoptosisinducing ligand

${ }^{*}$ Contributed equally

Key words: ovarian carcinoma, phosphatidylethanolamine binding protein, apoptosis, TRAIL, MAPK target, may be a promising approach for the treatment of ovarian cancer.

\section{Introduction}

Ovarian cancer is the most common gynecological malignancy worldwide; yet, the 5-year survival rate for this disease has remained low at $30 \%$ for the last 20 years with relatively little recent improvement (1). Despite initial tumor response rates of $80 \%$ to front-line platinum-based chemotherapy (2), the majority of patients with advanced ovarian cancer will ultimately have cancer relapse and develop drug resistance $(3,4)$. Drug resistance is a major problem in the clinical treatment of ovarian cancer, thus requiring new strategies to make ovarian cancer more sensitive to therapeutic agents (4).

Based on the facts that apoptosis and anti-apoptosis pathways are deeply related to drug sensitivity and resistance, treatment protocols aimed at apoptosis induction have been outlined. Tumor necrosis factor-related apoptosis-inducing ligand (TRAIL) is well known to trigger apoptosis in many malignant cells. Whereas cancer cells are responsive to TRAIL-induced cell death when used alone or in combination with other agents, normal cells are known to be relatively less sensitive to TRAIL, making it a desirable therapeutic agent to target a variety of cancers $(5,6)$. The potential and safety of soluble TRAIL as an anticancer therapeutic agent has been demonstrated in mice and non-human primates $(6,7)$. It has been reported that the combination of chemotherapy and TRAIL could induce apoptosis in some chemoresistant and TRAIL-resistant ovarian cancer cells (8).

hPEBP4 is a novel member of the phosphatidylethanolamine-binding protein (PEBP) family identified as an antiapoptotic molecule by us (9), and selectively expressed in breast cancer tissue. Silencing of hPEBP4 potentiates TNF $\alpha$ induced apoptosis and cell arrest in MCF-7 cells (10). Considering the preliminary data showing high expression of hPEBP4 in CaoV-3 ovarian cancer cells, in the present study, we investigated whether down-regulation of hPEBP4 expression in the CaoV-3 cells can sensitize the cells to TRAIL-induced apoptosis. Our results demonstrate that 


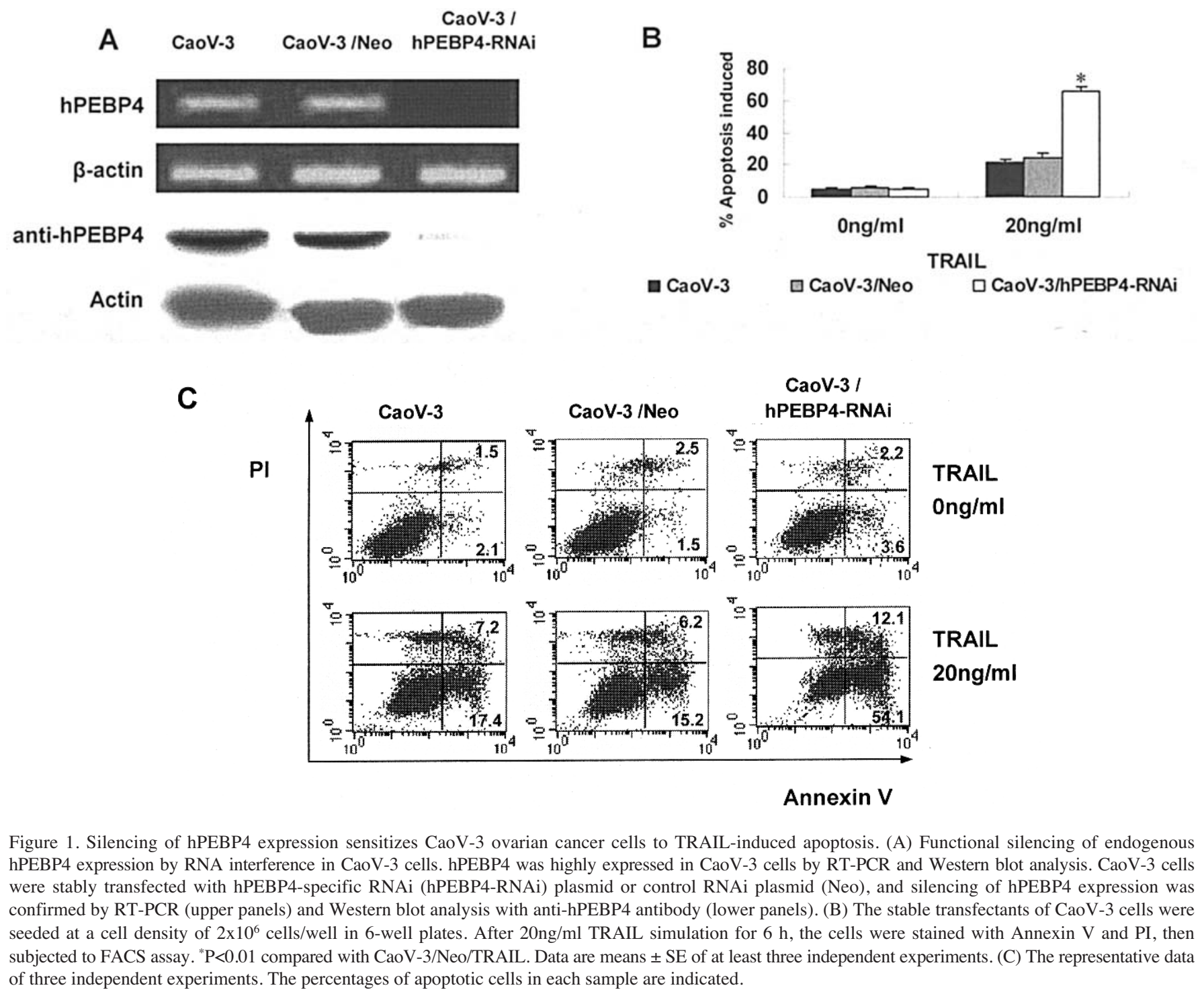

silencing of hPEBP4 significantly promotes TRAIL-induced apoptosis in CaoV-3 cells by activating ERK and JNK pathways. Therefore, down-regulation of anti-apoptotic hPEBP4 might represent a promising approach for the treatment of ovarian cancers.

\section{Materials and methods}

Reagents and cell culture. MEK1 inhibitor, PD98059, and JNK inhibitor, SP600125, were obtained from New England Biolabs and Calbiochem, respectively. TRAIL was purchased from PeproTech EC Ltd. Ovarian carcinoma CaoV-3 cells were grown in RPMI-1640 or DMEM supplemented with $10 \%(\mathrm{v} / \mathrm{v})$ fetal calf serum, $4.5 \mathrm{~g} / \mathrm{l} \mathrm{D}$-glucose, nonessential amino acids (100 $\mu \mathrm{M}$ each), 100 units/ml penicillin, $100 \mu \mathrm{g} / \mathrm{ml}$ streptomycin, and $2 \mathrm{mM}$ glutamine at $37^{\circ} \mathrm{C}$ in a $5 \% \mathrm{CO}_{2}$ atmosphere.

Generation of siRNA plasmid vector. IMG-800 (pSuppressorNeo, Imgenex, CA) vector was used for construction of 21-bp head-to-head hairpins of hPEBP4. The single-stranded oligonucleotides specific to hPEBP4, 5'-TCGAGGGAAA AGTCATCTCTCTCCTTgagtactgAAGGAGAGAGA
TGACTTTTCCCTTTTT-3' (sense) and 5'-CTAGAAAAA GGGAAAAGTCATCTCTCTCCTT cagtactcAAGGAG AGAGATGACTTTTCCC-3' (antisense), were synthesized and annealed to generate double-stranded DNAs, which were cloned into the $\mathrm{SalI}$ and $\mathrm{XbaI}$ cloning sites of pSuppressor/Neo (Imgenex). The plasmid construct (hPEBP4-RNAi) was then confirmed by sequencing. The control plasmid, Neo, contains a scrambled sequence that does not show significant homology to rat, mouse or human gene sequences (Imgenex).

Cell transfection and stable selection. The day before transfection, ovarian carcinoma CaoV-3 cells were plated in a 24well plate. The cells were $60-80 \%$ confluent at the time of transfection. hPEBP4-RNAi or Neo plasmid was transfected into $\mathrm{CaoV}-3$ cells using LipofectAMINE reagent (Invitrogen). Forty-eight hours after transfection, the cells were screened under $0.8 \mathrm{mg} / \mathrm{ml} \mathrm{G} 418$ (Merck) for 25 days. Clones of the stably transfected cells with Neo or hPEBP4-RNAi plasmid were obtained by limited dilution, designated as $\mathrm{CaoV}-3 / \mathrm{Neo}$ and CaoV-3/hPEBP4-RNAi, respectively.

Apoptosis assay. Cells were washed, resuspended in the staining buffer, and examined by ApoAlert Annexin V 


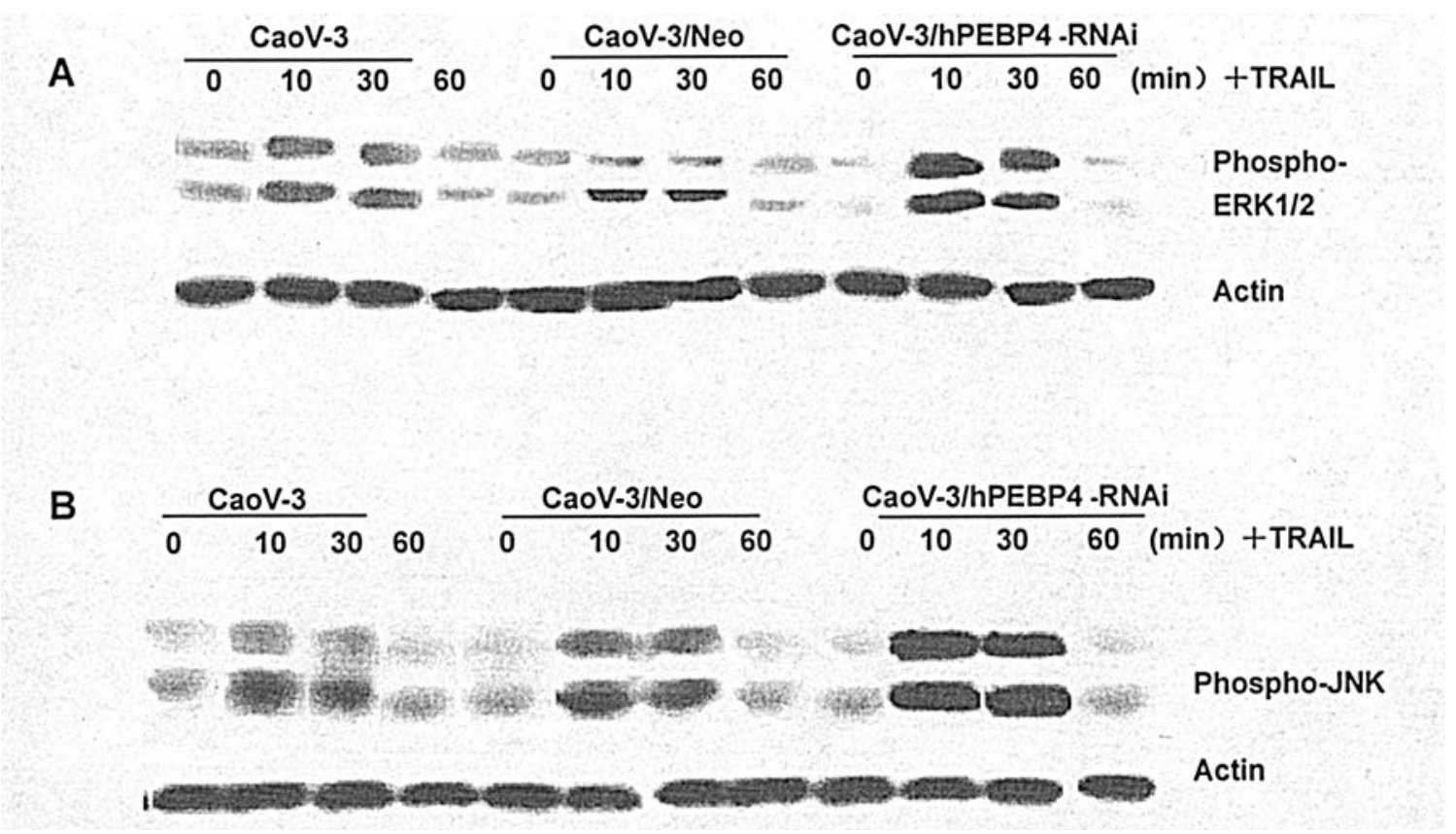

Figure 2. Silencing of hPEBP4 expression promotes TRAIL-induced MAPK activation in CaoV-3 ovarian cancer cells. (A) The effect of hPEBP4 silencing on TRAIL-induced activation of ERK1/2, MEK1 in CaoV-3 cells. The stable transfectants of CaoV-3 cells were serum-starved for $24 \mathrm{~h}$ and then treated with $10 \mathrm{ng} / \mathrm{ml}$ TRAIL for the indicated time. Cell lysates were subjected to immunoblotting analysis using anti-phospho-ERK1/2. (B) hPEBP4 RNA interference enhanced the activation of JNK induced by TRAIL.

apoptosis kit (Becton-Dickinson) or R123 (R-302, Molecular Probes) and PI according to the manufacturer's instructions, as described previously (10). Stained cells were analyzed by FACS (FACScalibur, Becton-Dickinson).

Co-immunoprecipitation assay. CaoV-3 lysates were precleared by protein A-sepharose beads (Sigma) for $1 \mathrm{~h}$ at $4^{\circ} \mathrm{C}$. Supernatants were collected and incubated with anti-hPEBP4 polyclonal antibody and protein A-Sepharose beads overnight at $4^{\circ} \mathrm{C}$. Immune complex beads were washed thoroughly with lysis buffer. Sample buffer $(50 \mu 1)$ was added to the pellets and the proteins were released from the beads by boiling for 5 min. Samples were subjected to Western blot analysis.

Western blot analysis. A BCA protein assay reagent kit (Pierce) was used to measure protein concentration. Samples containing equal amounts of protein were separated by $12 \%$ SDS-PAGE and transferred to Protran nitrocellulose membranes (Schleicher $\&$ Schuell). Blots were probed with antibodies specific for phospho-ERK1/2, phospho-MEK-1, phospho-Raf-1, phosphoJNK1/2, Raf-1, and MEK-1 (Santa Cruz), with appropriate horseradish peroxidase-conjugated antibodies as secondary antibodies (Cell Signaling). Supersignal West Femto maximum sensitivity substrate (Pierce) was used for the chemiluminescent visualization of proteins.

Statistical analysis. Statistical analysis (Fisher's exact test) was performed using the computer program SPSS Version 6.1.

\section{Results}

hPEBP4 is highly expressed in CaoV-3 ovarian cancer cells. $\mathrm{hPEBP} 4$ was found to promote cellular resistance to $\mathrm{TNF} \alpha-$ induced apoptosis, and down-regulation of hPEBP4 expression sensitizes MCF-7 breast cancer cells to TNF $\alpha$-induced apoptosis (9). We also observed that hPEBP4 is highly expressed in CaoV-3 ovarian cancer cells detected by RT-PCR and Western blot analysis (Fig. 1A). Because down-regulation of hPEBP4 expression in MCF-7 cells could enhance apoptosis sensitization and hPEBP4 is also highly expressed in CaoV-3 cells, we investigated whether or not silencing of hPEBP4 in CaoV-3 cells will promote the cellular apoptosis of CaoV-3 cells induced by TRAIL, which has been shown to be a potential agent to treat ovarian cancer.

Silencing of hPEBP4 expression sensitizes CaoV-3 ovarian cancer cells to TRAIL-induced apoptosis. It was reported that the combination of TRAIL and chemotherapeutic agents resulted in a significant increase in apoptosis in ovarian cancer cells (8). To evaluate the effect of hPEBP4 silencing on the sensitivity of CaoV-3 ovarian cancer cells to TRAIL-induced apoptosis, CaoV-3 cells were stably transfected with hPEBP4RNAi or Neo plasmids. Western blotting and RT-PCR confirmed the silencing of hPEBP4 expression in CaoV-3 stable transfectants (Fig. 1A). The transfectants were treated with $20 \mathrm{ng} / \mathrm{ml}$ TRAIL for $6 \mathrm{~h}$ and stained with Annexin V/PI. Annexin V-positive (including $\mathrm{PI}^{-}$or $\mathrm{PI}^{+}$) present apoptotic cells. As shown in Fig. 1B and C, 25\% of CaoV-3 cells and $21 \%$ of $\mathrm{CaoV}-3 / \mathrm{Neo}$ transfectants exhibited apoptosis respectively, when exposed to TRAIL. However, $66 \%$ of CaoV-3/hPEBP4-RNAi transfectants exhibited apoptosis. In addition, silencing of hPEBP4 in CaoV-3 cells did not affect the cell viability when compared to $\mathrm{CaoV}-3 / \mathrm{Neo}$ or the parental cells. This result suggested that silencing of hPEBP4 expression in CaoV-3 cells might contribute to an increased sensitivity to TRAIL-induced apoptosis. 


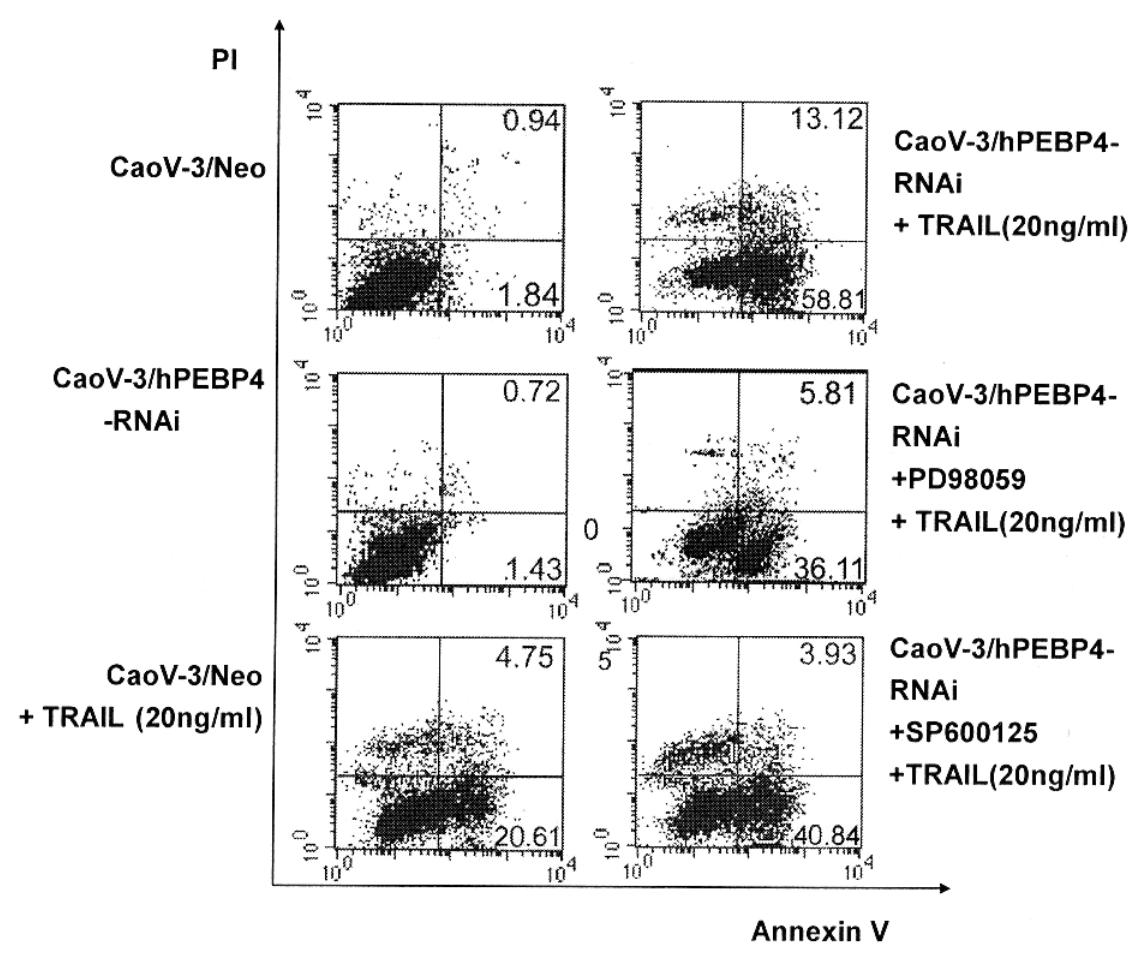

Figure 3. Enhanced ERK1/2 and JNK activation by hPEBP4 silencing in CaoV-3 cells potentiates TRAIL-induced apoptosis of CaoV-3 cells. PD98059 (MEK1 inhibitor) and SP600125 (JNK inhibitor) significantly decreased TRAIL-induced apoptosis in hPEBP4-silenced CaoV-3 cells. The stable transfectants of CaoV-3 cells were pre-incubated with $10 \mu \mathrm{M}$ PD98059 for $30 \mathrm{~min}$ or $20 \mu \mathrm{M} \mathrm{SP} 600125$ for $1 \mathrm{~h}$ at $37^{\circ} \mathrm{C}$ and subsequently stimulated with $20 \mathrm{ng} / \mathrm{ml}$ TRAIL for $6 \mathrm{~h}$. The cells were stained with Annexin V and PI, then subjected to FACS assay. The percentages of apoptotic cells in each sample are indicated.

Silencing of hPEBP4 expression in CaoV-3 cells potentiates TRAIL-induced MAPK activation. Next, we aimed to elucidate the mechanisms underlying the sensitization of $\mathrm{CaoV}-3$ cells to TRAIL-induced apoptosis by hPEBP4 silencing. Our previous study showed that TNF $\alpha$ induces hPEBP4 transfer from lysosomes to the cell membrane; where hPEBP4 binds to Raf-1 and MEK1, thus inhibiting MAPK activation (9). Because TRAIL-induced apoptosis involved MAPK activation (11-15), it was necessary to observe the effect of hPEBP4 silencing on TRAIL-induced MAPK activation. The stable transfectants of CaoV-3 cells were serum-starved for $24 \mathrm{~h}$ and treated with $10 \mathrm{ng} / \mathrm{ml}$ TRAIL for different time periods. As determined by Western blot assays, TRAIL stimulated ERK1/2 and JNK1 activity, which occurred 10 min after treatment and returned to baseline at $1 \mathrm{~h}$ (Fig. 2). Interestingly, silencing of hPEBP4 expression in CaoV-3 cells significantly enhanced TRAIL-induced activation of ERK1/2 and JNK1 (Fig. 2).

Inhibition of MAPK pathway reduces the increased TRAILinduced apoptosis in CaoV-3 cells by hPEBP4 silencing. TRAIL induces apoptosis in malignant cells by interacting with the DRs, including DR4 and DR5 (16). These receptors mediate the apoptotic signal through interaction of their intracellular death domains with adaptor proteins. It has been demonstrated that while TRAIL induces apoptosis, it also activates JNK and p44/42ERK1/2 (11-15). The roles of MAPK activation in apoptosis are highly controversial, with reports suggesting pro-apoptotic, anti-apoptotic and neutral roles (17-19). Since silencing of hPEBP4 expression in CaoV-3 cells significantly enhanced TRAIL-induced apoptosis and MAPK activation, we wondered whether the increased TRAIL-induced apoptosis might, in part, be attributable to an increased MAPK activity by hPEBP4 silencing. Therefore, we pre-incubated hPEBP4-silenced CaoV-3 cells with MEK1 inhibitor (PD98059) for $30 \mathrm{~min}$ or $20 \mu \mathrm{M}$ JNK inhibitor (SP600125) (20) for $1 \mathrm{~h}$ at $37^{\circ} \mathrm{C}$, then treated the cells with TRAIL. As shown in Fig. 3, pre-treatment with PD98059 or SP600125 significantly decreased the apoptosis of hPEBP4-silenced CaoV-3 cells induced by TRAIL. The apoptotic percentage decreased from $71.8 \%$ in CaoV-3/hPEBP4-RNAi cells to $41.9 \%$ after PD98059 treatment, or to $44.7 \%$ after SP600125 treatment, respectively. The data indicated that the sensitization of hPEBP4-silenced MCF-7 cells to TRAIL-induced apoptosis is due to the potentiated activation of the Raf-1/MEK/ERK pathway and JNK following TRAIL treatment.

Endogenous hPEBP4 protein associates with Raf-1 or MEK1 in CaoV-3 cells upon TRAIL treatment. Our previous study demonstrated that hPEBP4 binds to Raf-1 and MEK1 following TNF $\alpha$ treatment, thus inhibiting downstream MEK1/ERK activation (9). Since the above experiments proved that hPEBP4 was involved in TRAIL-induced ERK1/2 activation, we examined the interaction of endogenous hPEBP4 with Raf-1 and MEK1 in CaoV-3 cells upon TRAIL treatment. Anti-hPEBP4 polyclonal antibody was used to immunoprecipitate endogenous hPEBP4 from $\mathrm{CaoV}-3$ cells stimulated with or without TRAIL. As shown in Fig. 4, the presence of Raf-1 and MEK1 was detected in the anti-hPEBP4 immunoprecipitates of CaoV-3 cells treated with TRAIL, but not in those of unstimulated cells. The results suggested that hPEBP4 binds to Raf-1 or MEK-1 upon TRAIL treatment, which might dissociate the Raf-1-MEK complex, and thus functions as a 

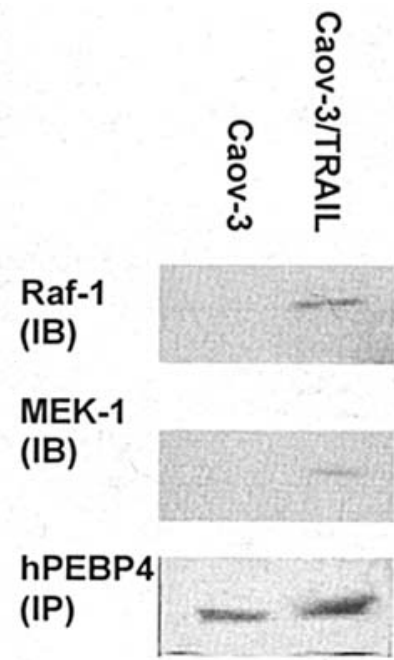

Figure 4. Endogenous hPEBP4 protein associates with Raf-1 or MEK1 in CaoV-3 cells following TRAIL treatment. Lysates of CaoV-3 cells treated with or without $10 \mathrm{ng} / \mathrm{ml}$ TRAIL for $10 \mathrm{~min}$ were pre-cleared by protein Asepharose beads (Sigma) for $1 \mathrm{~h}$ at $4^{\circ} \mathrm{C}$. Supernatants were collected and incubated with anti-hPEBP4 polyclonal antibody cross-linked to protein-A Sepharose beads overnight at $4^{\circ} \mathrm{C}$. Immune complex beads were washed thoroughly with lysis buffer. Sample buffer $(50 \mu 1)$ was added to the pellets and the proteins were released from the beads by boiling for $5 \mathrm{~min}$. Samples were immunoblotted using Raf-1 or MEK1 or anti-hPEBP4 antibody.

competitive inhibitor of MEK phosphorylation. Therefore, silencing of hPEBP4 expression potentiates TRAIL-induced activation of the Ras/Raf-1/MEK/ERK signaling pathway, which contributes to the increased sensitivity of CaoV-3 cells to TRAIL-induced apoptosis.

\section{Discussion}

The current therapeutic approach is not very effective in treating ovarian cancer patients $(21,22-24)$. Therefore, more efficient treatment protocols aimed at different targets need to be explored. Inducers of apoptosis have been applied in cancer treatment; however, chemotherapy or radiation is not invariably cytotoxic to all cancer cells. It has been observed that defects in the apoptotic pathway in cancer cells confer insensitivity to the cytotoxic effects of chemotherapy and may therefore represent an important mechanism for cancer cell drug resistance $(25,26)$. Several studies have attempted to induce cancer cell apoptosis by targeting or silencing the anti-apoptotic proteins, and have shown promising results $(27,28)$. Enhancing the chemosensitivity of cancer cells by the transfer or interference of genes that influence the death and growth of the cell is one of the most important strategies in cancer therapeutics.

The TRAIL or Apo2 ligand is a member of the TNF superfamily of cell death-inducing ligands (29). The TRAILmediated apoptosis signal pathway consists of activated TRAIL-R1/R2, recruited Fas-associated death domain protein (FADD). TRAIL-R1 or -R2 can also activate MAPK signaling pathways and the activation is required for the sensitization of PC3 cells to TRAIL-induced apoptosis $(30,31)$. Our previous study demonstrated that hPEBP4 could inhibit TNF $\alpha$-induced apoptosis (9). In this study we found that silencing of hPEBP4 expression in CaoV-3 cells promoted TRAIL-induced apoptosis. Simultaneously, silencing of hPEBP4 in CaoV-3 cells enhanced TRAIL-induced ERK and JNK activation. In addition, the increased TRAIL sensitivity induced by hPEBP4 silencing was partially reduced by PD98059 or SP600125, the inhibitors of ERK and JNK activation, respectively. Thus, our results suggest that ERK and JNK might act under certain circumstances in a pro-apoptotic fashion. Similar data have been reported for cisplatin-induced apoptosis and TRAILinduced apoptosis (17-19). In this study, endogenous hPEBP4 association with both MEK1 and Raf-1 after TRAIL stimulation was observed using co-immunoprecipitation assays, confirming our assumption that hPEBP4 binds to Raf-1 or MEK1 upon TRAIL stimulation, thus dissociating the Raf-1-MEK complex, and inhibiting MEK/ERK phosphorylation. The results may explain why TRAIL-induced activation of the Ras/Raf-1/MEK1/ERK signaling pathway was further enhanced in hPEBP4-silenced cells.

Besides abundant expression in ovarian carcinoma cells, we also detected the high expression of hPEBP4 in breast and prostate cancer cells. In addition to the CaoV-3 cell model, silencing of hPEBP4 expression rendered other cancer cells, which highly express hPEBP4 such as MCF-7 cells, more sensitive to TNFa-induced cell death $(9,10)$. hPEBP4 could be particularly important if the point at which it exerts an effect is common to apoptosis induced by other anti-cancer agents. By decreasing hPEBP4 expression in breast and ovarian cancer cells, the threshold at which chemotherapeutic agents trigger cancerous cells to undergo apoptosis may be lowered, leading to a more favorable response to chemotherapeutic agents. Therefore, its high expression in some cancers and its ability to function as an anti-apoptotic molecule outline that $\mathrm{hPEBP} 4$ is a promising target to be silenced for the treatment of cancers. Down-regulating hPBPP4 may prove to be an effective treatment for cancers that highly express hPBPP4, such as ovarian, prostate and breast carcinoma.

\section{Acknowledgements}

This work was supported by grants from the National Natural Science Foundation of China (30121002), the National Key Basic Research Program of China (2001CB510002) and the National High Biotechnology Development Program of China (2002BA711A01).

\section{References}

1. Cannistra SA: Cancer of the ovary. N Engl J Med 351: 2519-2529, 2004

2. Sifri R, Gangadharappa S and Acheson LS: Identifying and testing for hereditary susceptibility to common cancers. CA Cancer J Clin 54: 309-326, 2004.

3. Barnes MN, Grizzle WE, Grubbs CJ and Partridge EE: Paradigms for primary prevention of ovarian carcinoma. CA Cancer J Clin 52: 216-225, 2002.

4. Lehnert M: Clinical multidrug resistance in cancer: a multifactorial problem. Eur J Cancer 32A: 912-920, 1996.

5. Ashkenazi A and Dixit V: Death receptors: signaling and modulation. Science 281: 1305-1308, 1998.

6. Ashkenazi A, Pai RC, Fong S, et al: Safety and anti-tumor activity of recombinant soluble Apo2 ligand. J Clin Invest 104: 155-162, 1999.

7. Walczak H, Miller RE, Ariail K, et al: Tumoricidal activity of tumor necrosis factor-related apoptosis-inducing ligand in vivo. Nat Med 5: 157-163, 1999. 
8. Cuello M, Ettenberg SA, Nau MM and Lipkowitz S: Synergistic induction of apoptosis by the combination of TRAIL and chemotherapy in chemoresistant ovarian cancer cells. Gynecol Oncol 81: 380-390, 2001.

9. Wang X, Li N, Liu B, et al: A novel human phosphatidylethanolamine-binding protein resists $\mathrm{TNF} \alpha$-induced apoptosis by inhibiting mitogen-activated protein kinase pathway activation and phosphatidylethanolamine externalization. J Biol Chem 279: 45855-45864, 2004.

10. Wang X, Li N, Li H, et al: Silencing of human phosphatidylethanolamine-binding protein 4 sensitizes breast cancer cells to $\mathrm{TNF} \alpha$-induced apoptosis and cell growth arrest. Clin Cancer Res 11: 7545-7553, 2005.

11. Lin Y, Devin A, Cook A, et al: The death domain kinase RIP is essential for TRAIL (Apo2L)-induced activation of IkappaB kinase and c-Jun N-terminal kinase. Mol Cell Biol 20: 6638-6645, 2000.

12. Wen L, Zhuang L, Luo X and Wei P: TL1A-induced NF-kappaB activation and c-IAP2 production prevent DR3-mediated apoptosis in TF-1 cells. J Biol Chem 278: 39251-39258, 2003.

13. Tran SE, Holmstrom TH, Ahonen M, Kahari VM and Eriksson JE: MAPK/ERK overrides the apoptotic signaling from Fas, TNF, and TRAIL receptors. J Biol Chem 276: 16484-16490, 2001.

14. Ohtsuka T and Zhou T: Bisindolylmaleimide VIII enhances DR5mediated apoptosis through the MKK4/JNK/p38 kinase and the mitochondrial pathways. J Biol Chem 277: 29294-29303, 2002.

15. Samanta AK, Huang HJ, Bast RC Jr and Liao WS: Overexpression of MEKK3 confers resistance to apoptosis through activation of NFkappaB. J Biol Chem 279: 7576-7583, 2004.

16. Wang S and El-Deiry WS: TRAIL and apoptosis induction by TNF-family death receptors. Oncogene 22: 8628-8633, 2003.

17. Christ O, Seiter S, Matzku S, Burger C and Zoller M: Efficacy of local versus systemic application of antibody-cytokine fusion proteins in tumor therapy. Clin Cancer Res 7: 985-998, 2001

18. Bergmann A, Agapite J, McCall K and Steller H: The Drosophila gene hid is a direct molecular target of Rasdependent survival signaling. Cell 95: 331-341, 1998.

19. Tang G, Minemoto Y, Dibling B: Inhibition of JNK activation through NF-kappaB target genes. Nature 414: 313-317, 2001

20. Han Z, Boyle DL, Chang L, et al: c-Jun N-terminal kinase is required for metalloproteinase expression and joint destruction in inflammatory arthritis. J Clin Invest 108: 73-81, 2001.
21. Blank SV, Chang R, Muggia F, et al: Epidermal growth factor receptor inhibitors for the treatment of epithelial ovarian cancer. Oncology 19: 553-559, 2005.

22. Santin AD, Cane S, Bellone S, et al: Treatment of chemotherapy-resistant human ovarian cancer xenografts in C.B-17/ SCID mice by intraperitoneal administration of Clostridium perfringens enterotoxin. Cancer Res 65: 4334-4342, 2005.

23. Berchuck A, Iversen ES, Lancaster JM, et al: Patterns of gene expression that characterize long-term survival in advanced stage serous ovarian cancers. Clin Cancer Res 11: 3686-3696, 2005.

24. Chan JK, Pham H, You XJ, et al: Suppression of ovarian cancer cell tumorigenicity and evasion of cisplatin resistance using a truncated epidermal growth factor receptor in a rat model. Cancer Res 65: 3243-3248, 2005.

25. Zornig M, Hueber A, Baum W and Evan G: Apoptosis regulators and their role in tumorigenesis. Biochim Biophys Acta 1551: F1-F37, 2001.

26. Ibrado AM, Liu L and Bhalla K: Bcl-xL overexpression inhibits progression of molecular events leading to paclitaxel-induced apoptosis of human acute myeloid leukemia HL-60 cells. Cancer Res 57: 1109-1115, 1997.

27. Wall NR and Shi Y: Small RNA: can RNA interference be exploited for therapy? Lancet 362: 1401-1403, 2003.

28. Trougakos IP, So A, Jansen B, et al: Silencing expression of the clusterin/apolipoprotein $\mathrm{j}$ gene in human cancer cells using small interfering RNA induces spontaneous apoptosis, reduced growth ability, and cell sensitization to genotoxic and oxidative stress. Cancer Res 64: 1834-1842, 2004.

29. Wiley SR, Schooley K, Smolak PJ, et al: Identification and characterization of a new member of the TNF family that induces apoptosis. Immunity 3: 673-682, 1995.

30. Wen-Hui H, Holly J and Hong-Bing S: Tumor necrosis factorrelated apoptosis-inducing ligand receptors signal NF-кB and JNK activation and apoptosis through distinct pathways. J Biol Chem 274: 30603-30610, 1999.

31. Sah NK, Munshi A, Kurland JF, et al: Translation inhibitors sensitize prostate cancer cells to apoptosis induced by tumor necrosis factor-related apoptosis-inducing ligand (TRAIL) by activating c-Jun N-terminal kinase. J Biol Chem 278: 20593-20602, 2003. 